\title{
DIAGNOSTIC EFFICACY OF LIGHT-INDUCED FLUORESCENCE METHOD AND DIGITAL FIBER-OPTIC TRANSILLUMINATION IN THE ASSESSMENT OF EARLY CARIES IN CHILDREN
}

\author{
Magdalena Prudel-Babiuch, Lidia Postek-Stefańska, Jarosław Babiuch \\ Department of Pediatric Dentistry, Faculty of Medical Sciences in Zabrze, Medical University of Silesia in Katowice, Poland
}

\begin{abstract}
INTRODUCTION: New diagnostic devices' development is very important to detect caries lesions at earliest stage. The main aim of the study was the assessment of new dental caries detection methods: digital fiber-optic transillumination (DIFOTI) and quantitative light-induced fluorescence (QLF), in comparison with conventional method: visual and tactile examination.

OвJECTIVEs: Specific aims were the comparison of efficacy of diagnostic devices, including DIAGNOcam (DIFOTI), DIAGNOdent pen, and VistaCam iX (QLF) in early caries detection in children, and the assessment of their sensitivity and specificity.

MATERIAL AND METHODs: In total, 61 children, aged 5-18 were examined. Early caries (caries incipiens) on occlusal and approximal surfaces of molar deciduous, and permanent teeth and premolar teeth were diagnosed. Examination scheme included visual and tactile examination according to ICDAS-II (international caries detection and assessment system), and assessment of dental caries with VistaCam iX, DIAGNOdent pen, and DIAGNOcam devices.

RESULTS: 227 surfaces were examined, including 129 permanent molars' surfaces, 50 deciduous molars' surfaces, and 48 premolars' surfaces. Therefore in total, 172 occlusal surfaces and 55 approximal surfaces were analyzed. The highest sensitivity was obtained with VistaCam iX -0.9655 , lower DIAGNOcam -0.8649 , and DIAGNOdent pen -0.6410 . The highest specificity was obtained with DIAGNOdent pen -0.9043 , lower VistaCam iX -0.9028 , and DIAGNOcam - 0.8947 .

ConcLusions: The clinical visual and tactile examination should be the basic diagnostic method, and their results should be confirmed with additional devices, such as VistaCam iX, DIAGNOcam, or DIAGNOdent pen. None of the mentioned modern devices provided total efficacy in early caries detection. VistaCam iX and DIAGNOcam obtained better sensitivity than DIAGNOdent pen; however, all devices achieved high specificity.
\end{abstract}

KEY WORDS: laser fluorescence, transillumination, dental caries detection, DIAGNOdent, fluorescence camera.

J Stoma 2021; 74, 2: 101-109

DOI: https://doi.org/10.5114/jos.2021.106499

\section{JOURNAL OF} STOMATOLOGY CZASOPISMO STOMATOLOGICZNE

AdDress FOR CORRESPONDENCE: Dr. Magdalena Prudel-Babiuch, Department of Pediatric Dentistry, Faculty of Medical Sciences in Zabrze, Medical University of Silesia in Katowice, Poland, e-mail:magdaprudel@gmail.com

ReCEIVED: 19.11 .2020 • ACCePted: 10.03.2021 • Published: 04.06.2021 


\section{INTRODUCTION}

Dentists use the visual and tactile examination and radiograms to detect and diagnose carious lesions. Contemporary dental diagnostics opens new possibilities, thanks to the appearance of modern diagnostic devices. The specificity of the visual method, in other words, the ability to detect healthy teeth, has been reported in literature at a level of $90 \%$; however, its sensitivity, i.e., the ability to detect teeth with caries, at a level between $12 \%$ and $82 \%$. These divergencies may be due to using different caries classification systems and differences in detecting caries in hard-to-reach areas [1]. Reproducibility of the visual method is low, due to examiner's subjectivity of measurement [2]. In order to standardize the caries diagnostics system, in 2001, international caries detection and assessment system ICDAS-I was introduced. The criteria of ICDAS have been evaluated in terms of correlation with the results obtained after histopathological examination of extracted teeth. The system is applicable in the examination of dental crowns caries, without referring to root caries, or the caries progress level [3]. In 2005, the international caries detection and assessment system ICDAS-II was introduced (Table 1).

Both the conventional and digital radiography are used by clinicians to complete clinical examination. These methods show very high sensitivity in detecting dentin caries of proximal surfaces; however, their utility in early enamel decay detection is limited [4]. In case of occlusal caries detection on bitewing X-ray, the cavity may extend to central one-third part of the dentin, which excludes the possibility of providing non-invasive treatment [5]. Modern methods of early caries diagnostics based on the phenomenon of fluorescence, include quantitative light-induced fluorescence (QLF) and its diagnostic devices, such as DIAGNOdent, DIAGNOdent pen, VistaProof camera, and VistaCam iX. One of the most widely featured diagnostic devices is the DIAGNOdent 2095 (KaVo, Biberach, Germany), designed for the purpose of diagnostics of early caries

TABLE 1. ICDAS-\| codes

\begin{tabular}{|l|l|}
\hline Code & Description \\
\hline 0 & Sound enamel \\
\hline 1 & White/brown spot in dry enamel \\
\hline 2 & White/brown spot in wet enamel \\
\hline 3 & $\begin{array}{l}\text { Localized enamel breakdown (without clinical visual signs } \\
\text { of dentinal involvement) }\end{array}$ \\
\hline 4 & $\begin{array}{l}\text { Underlying dark shadow from dentin with or without localized } \\
\text { enamel breakdown }\end{array}$ \\
\hline 5 & Distinct cavity with visible dentin \\
\hline 6 & Extensive distinct cavity with visible dentin \\
\hline
\end{tabular}

of both occlusal and smooth surfaces, which was introduced to medical market in 1998.

In 2005, the DIAGNOdent pen (DIAGNOdent 2190, KaVo, Biberach, Germany) was launched, which also facilitated detecting caries on proximal surfaces and analyzing bacterial plaque in the gingival pockets. The most modern systems for caries detection are the intraoral cameras, equipped in light-emitting diode (LED). They include fluorescent camera VistaProof (Dürr Dental SE, Germany) brought to the medical market in 2007, and its modernized version VistaCam iX, introduced by the same company in 2011 as well as SoproLife camera [6-8]. Other modern techniques of caries diagnostics are based on the phenomenon of digital transillumination DIFOTI (digital fiber-optic trans-illumination). It uses non-visible infrared light, which passes through the examined surface that is focused by a system of lenses from charge-coupled device (CCD) camera, and the image is displayed on a computer screen. DIAGNOcam (KaVo, Biberach, Germany) is a device based on DIFOTI. Supported by modern devices, caries diagnostics precisely facilitates the exposition of early caries of both occlusal and proximal tooth surfaces, and may, at the same time, be an alternative to commonly used bitewing X-ray. The exact description of the stage of early carious lesion enables the implementation of non-invasive treatment, such as enamel remineralization, which may prevent the necessity of cavity preparation with the use of a drill and applying dental filling. In more advanced cases, it enables cavity preparation with a minimally invasive method. Most often, early carious lesions develop in hard and clean spaces, such as fissures, pits, and proximal surfaces of the teeth. Relatively quick detection of lesions enables the implementation of the best method of treatment, saving tooth tissue. It is particularly important in children, who develop caries lesions faster than adults.

\section{OBJECTIVES}

The aim of this study was the evaluation of modern caries diagnostics methods, i.e., digital fiber-optic transillumination (DIFOTI) and quantitative light-induced fluorescence (QLF) in comparison to the conventional method of visual and tactile examination. More specifically, its objective was to compare the efficacy of modern diagnostic devices, including the DIAGNOcam (DIFOTI), DIAGNOdent pen, and VistaCam iX (QLF) in the detection of early carious lesions in children as well as the evaluation of sensitivity and specificity of the respective methods with the application of DIAGNOcam, DIAGNOdent pen, and VistaCam iX camera.

\section{MATERIAL AND METHODS}

The study material was collected between 2013-2016 at the Pedodontics Clinic of Academic Center of Den- 
tistry and Specialized Medicine in Bytom, the Medical University of Silesia in Katowice, and the Non-Public Healthcare Center of Medicine and Dentistry in Rybnik. The study included 61 children aged 5-18, generally healthy, both sexes, whose parents gave consent for the tests. The diagnostics comprised of fully erupted molar deciduous and permanent teeth (excluding wisdom teeth) as well as premolars without fillings, fissure sealants, extrinsic and intrinsic discoloration, and enamel disorders, such as hypoplasia or hypomineralization. Early caries (caries incipiens) of occlusal and proximal surfaces of 224 teeth was the subject of diagnosis, and 227 teeth surfaces were examined. If carious lesion was detected on proximal and occlusal surfaces or both the occlusal surfaces of the tooth, it was treated as two separate units.

The material obtained from each patient included medical record of visual and tactile examination according to ICDAS-II, results from DIAGNOdent pen (KaVo, Biberach, Germany), and photos of the teeth made with the VistaCam iX (Dürr Dental SE, Bietigheim-Bissingen, Germany) and the DIAGNOcam (KaVo, Biberach, Germany). The assessment of the results was based on statistical analysis of data from the clinical examination ICDAS-II, the DIAGNOdent pen examination, and pictures taken with the VistaCam iX and DIAGNOcam.

\section{EXAMINATION SCHEME}

\section{CLINICAL EXAMINATION: VISUAL AND TACTILE ASSESSMENT}

The carious lesions of the tooth surface were put under visual and tactile evaluation after being cleaned from plaque with fluoride-free toothpaste and dried under artificial light of a dental unit, with the use of a flat mirror without magnifying or probe. The results were entered into the patient's examination card according to ICDAS-II (international caries detection and assessment system).

\section{VISTACAM IX EXAMINATION}

Carious lesions on occlusal surfaces were examined with the VistaCam iX. Two heads were used: CAM for imaging of the intraoral anatomical structures, and PROOF - for the detection of caries by means of fluorescence (QLF). Fluorescent light was registered by the camera's lens, sent to a computer, and analyzed by a specialistic DBSWIN software (Dürr, Germany). This led the photography to be seen on the monitor, which showed the decay in a yellow-red-green color scale with a corresponding numeric scale of $0-4$, characterizing the decay's depth in a given place.

The numeric scale of the camera's producer presents the fluorescence intensification as follows: 0.0-0.9 healthy enamel (green); > 0.9-1.5 early enamel caries (blue);
$>$ 1.5-2.0 enamel caries extended to dentin-enamel junction (red); > 2.0-2.5 dentin caries (orange); > 2.5 deep dentin caries (yellow) [9].

\section{CARIES DIAGNOSTICS WITH THE DIAGNODENT PEN}

Before the examination, the DIAGNOdent pen device based on the phenomenon of laser fluorescence, was calibrated by applying the probe to the ceramic model and to a healthy, smooth tooth surface. The A head was used on the occlusal surfaces, while the $\mathrm{B}$ head, on the proximal ones. The probe was being moved along the tooth and the highest score within 0-99 range was read and then interpreted according to the scale of Lussi et al. [10] as follows: 0-13 lack of active caries (professional tooth cleaning recommended); 14-20 caries of the external enamel layer (preventive action recommended); 21-29 caries extended to the internal enamel layer (preventive action or minimally invasive treatment recommended); $\geq 30$ dentine caries (minimally invasive treatment recommended).

\section{DIAGNOCAM EXAMINATION}

The DIAGNOcam camera uses the phenomenon of digital transillumination (DIFOTI). The head of the camera was being moved along the tooth surface and after obtaining the best image sharpness, a set of photographs was made, and then displayed on the computer's screen. The teeth's carious surfaces were shown in different planes as shadows of different saturation. So far, there is no numeric scale describing the caries range (depth) based on the grade of tooth's obscuration. For the purpose of this work, a percentage system was created, helping in the interpretation of the photos, based on the area that measured the curves in the tooth's photograph (the obscured area against the whole tooth surface) and described as follows: $0-0.2 \%$ : healthy enamel; $>0.2-2 \%$ : caries of the external enamel layer; > $2 \%$ : caries of the external and internal enamel layer. Figures 1-3 show example photos of the occlusal surface of tooth 46. The first photo (1) was taken with the CAM head, the second (2) with the PROOF head of VistaCam iX, and the third one (3) was taken with the DIAGNOcam. The oral cavity hygiene level of the examined children was also put under visual assessment, and was qualified as good, medium, or bad. The test unit was the surface of a deciduous or permanent tooth. The distribution of variables was characterized by group size $\mathrm{n}$ (the number or tooth surfaces), arithmetic mean, standard deviation $(M \pm S D)$, relative standard error, median, minimum, maximum, and level of confidence (95.0\%). The data obtained from the measurement were statistically analyzed using Statistica 10 software in Excel spreadsheet (Microsoft Office). 


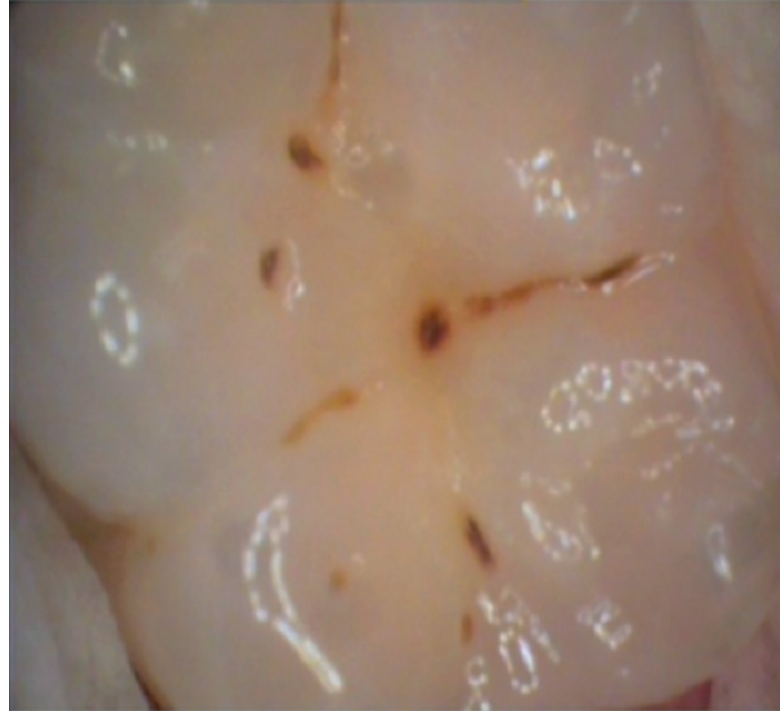

FIGURE 1. Photo image of the occlusal surface of tooth 46 taken with the CAM head of VistaCam iX

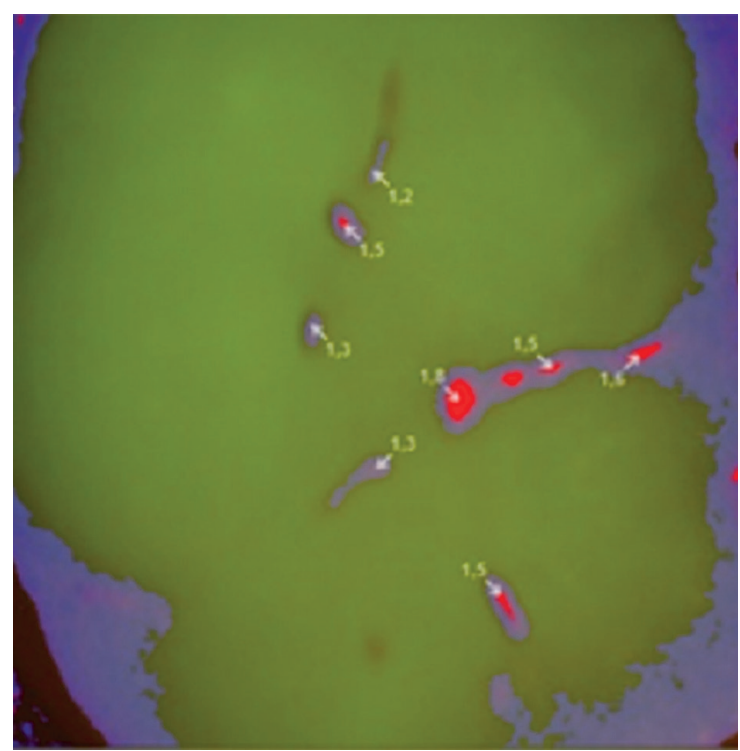

FIGURE 2. Photo image of the occlusal surface of tooth 46 taken with the PROOF head of VistaCam iX

\section{RESULTS}

Sixty-one patients aged between 5 and 18 (23 boys and 38 girls) were examined. In total, 224 teeth were evaluated, including 129 molar permanent teeth (129 surfaces), 45 premolars (48 surfaces), and 50 molar deciduous teeth (50 surfaces). Overall, 227 tooth surfaces were assessed, with 172 occlusal and 55 proximal surfaces (Table 2).

In the evaluation of the efficacy of diagnostic methods with the use of DIAGNOcam, DIAGNOdent pen, and VistaCam iX, the results obtained during the visual and tactile examination and expressed by codes of ICDAS-II were adopted as the basis. The results are

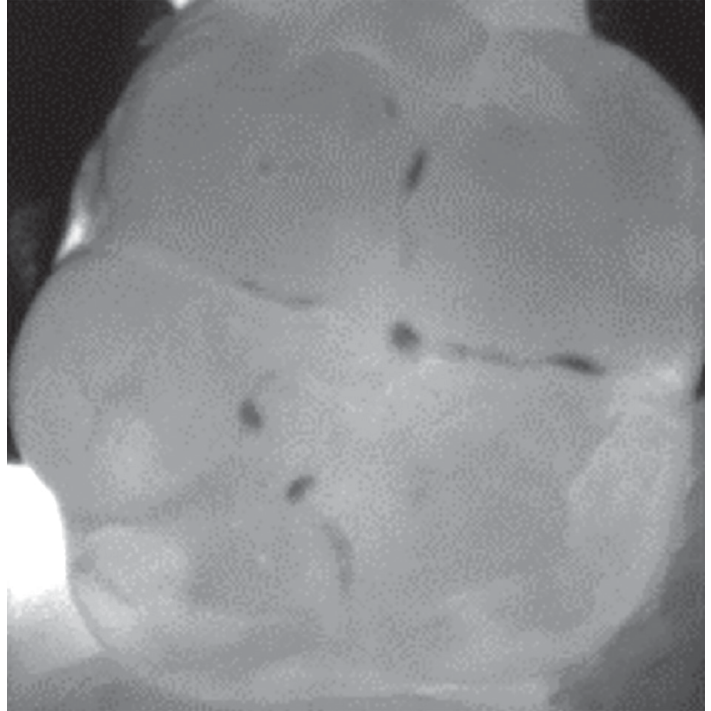

FIGURE 3. Photo image of the occlusal surface of tooth 46 taken with the DIAGNOcam

presented in Table 3. In the visual-tactile examinations, code 0 of ICDAS-II indicating healthy enamel was obtained on 27 tooth surfaces (11.89\%). 76 surfaces (33.48) obtained code 1 , signifying early enamel decay, and the majority of surfaces, 89 (39.21\%), was given code 2 proving enamel caries more extended than in case of code 1.35 surfaces (15.42\%) were classified as code 3 , showing local surface discontinuity without dentin changes. In the VistaCam iX examinations, the 0-0.9 result showing healthy enamel was obtained on 11 tooth surfaces $(6.36 \%)$. The $>0.9-1.5$ result showing early enamel caries was found on 88 tooth surfaces (50.87\%), and the $>1.5$ result showing enamel decay extended to dentin-enamel junction was obtained on 74 surfaces $(42.77 \%)$. In the DIAGNOdent pen examinations, the $0-13$ result, meaning lack of decay, was obtained on 63 tooth surfaces $(27.75 \%)$. The $14-20$ result, meaning caries of the external enamel layer, was obtained on 35 surfaces (15.42\%), and the 21-29 result, meaning more profound decay, extended to the internal enamel layer was obtained on 40 surfaces (16.62\%). In case of 89 tooth surfaces $(39.21 \%)$, the $\geq 30$ result was obtained, meaning dentine extended decay. The DIAGNOcam examinations, the $0-0.2 \%$ result signifying healthy enamel was obtained on 31 tooth surfaces $(13.66 \%)$. The $>0.2-2 \%$ result, meaning external enamel layer decay, was obtained on 97 tooth surfaces $(42.73 \%)$, and the $>2 \%$ result, meaning more profound external enamel layer decay, was obtained on 99 surfaces (43.61\%).

The highest sensitivity of the method amounting to 0.9655 was found with the VistaCam iX camera. The DIAGNOcam examination's sensitivity amounted to 0.8649 , and the DIAGNOdent pen analysis it was 0.6410 . All the devices showed respectively similar and high specificity. The highest specificity was obtained with 
TABLE 2. Number of examined group and teeth/ surfaces with division of dental groups and sex

\begin{tabular}{|c|c|c|c|c|c|c|c|c|c|c|c|}
\hline \multirow[b]{2}{*}{ 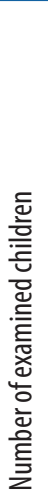 } & \multirow[b]{2}{*}{ 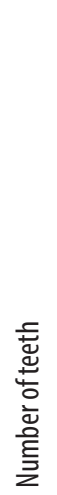 } & \multirow[b]{2}{*}{ 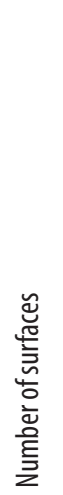 } & \multicolumn{3}{|c|}{ General } & \multicolumn{3}{|c|}{ Male } & \multicolumn{3}{|c|}{ Female } \\
\hline & & & 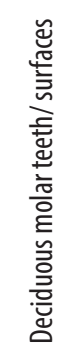 & 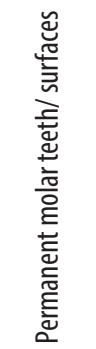 & 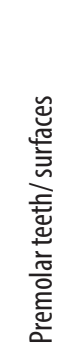 & 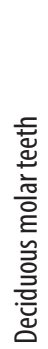 & 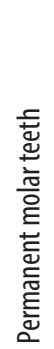 & 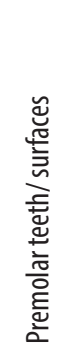 & 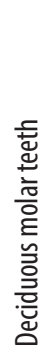 & 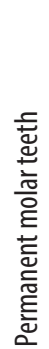 & 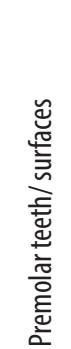 \\
\hline 61 & 224 & 227 & $50 / 50$ & $129 / 129$ & $45 / 48$ & 17 & 48 & $19 / 21$ & 33 & 81 & $26 / 27$ \\
\hline
\end{tabular}

TABLE 3. Assessment of initial caries made with VistaCam iX, DIAGNOdent pen, and DIAGNOcam

\begin{tabular}{|c|c|c|c|c|c|c|c|c|c|c|c|}
\hline \multicolumn{3}{|c|}{ Visual tactile method } & \multicolumn{3}{|c|}{ VistaCam iX } & \multicolumn{3}{|c|}{ DIAGNOdent pen } & \multicolumn{3}{|c|}{ DIAGNOcam } \\
\hline ICDAS-II & $n$ & $\%$ & Producent's scale & $n$ & $\%$ & Lussi's scale & $n$ & $\%$ & Authors'scale & $n$ & $\%$ \\
\hline 0 & 27 & 11.89 & $0-0.9$ & 11 & 6.36 & $0-13$ & 63 & 27.75 & $0-0.2 \%$ & 31 & 13.66 \\
\hline 1 & 76 & 33.48 & \multirow{2}{*}{$>0.9-1.5$} & \multirow{2}{*}{88} & \multirow{2}{*}{50.87} & \multirow{2}{*}{$14-20$} & \multirow{2}{*}{35} & \multirow{2}{*}{15.42} & \multirow{2}{*}{$>0.2-2 \%$} & \multirow{2}{*}{97} & \multirow{2}{*}{42.73} \\
\hline 2 & 89 & 39.21 & & & & & & & & & \\
\hline 3 & 35 & 15.42 & \multirow{2}{*}{$>1.5$} & \multirow{2}{*}{74} & \multirow{2}{*}{42.77} & $21-29$ & 40 & 16.62 & \multirow{2}{*}{$>2 \%$} & \multirow{2}{*}{99} & \multirow{2}{*}{43.61} \\
\hline$>3$ & 0 & 0 & & & & $\geq 30$ & 89 & 39.21 & & & \\
\hline All & 227 & - & All & 173 & - & All & 227 & - & All & 227 & - \\
\hline
\end{tabular}

the DIAGNOdent pen -0.9043 , followed by the VistaCam iX with 0.9028 , and the DIAGNOcam - 0.8947 (Table 4). This was confirmed by Spearman's rank correlation coefficient results. Comparing the results from individual devices in relation to those from the visual and tactile examination, ICDAS-II codes 1-3 showed practically full dependence; however, they showed no connection in case of comparing healthy surfaces (code 0 of ICDAS-II) (Table 5).

\section{DISCUSSION}

The development of modern diagnostic methods is extremely important in order to confirm clinical examination diagnosis and detect carious lesions as early as possible, and later to apply a non-invasive treatment. In literature, the largest number of papers on the usage and effectiveness of modern diagnostic devices refers to DIAGNOdent and its newer version, DIAGNOdent pen. Shi et al. compared the effectiveness of fluorescence method and the conventional bitewing photographs. They came to a conclusion that DIAGNOdent demonstrates higher efficacy in the enamel and dentin caries detection than X-rays [11]. Different results were obtained by Anges et al. who evaluated wisdom teeth before
TABLE 4. Sensitivity, specificity, and confidence intervals of the examined methods

\begin{tabular}{|c|c|c|c|}
\hline & VistaCam iX & DIAGNOdent pen & DIAGNOcam \\
\hline Sensitivity & 0.9655 & 0.6410 & 0.8649 \\
\hline$-95 \% \mathrm{Cl}$ & 0.8224 & 0.4718 & 0.7123 \\
\hline$+95 \% \mathrm{Cl}$ & 0.9992 & 0.7880 & 0.9546 \\
\hline Specificity & 0.9028 & 0.9043 & 0.8947 \\
\hline$-95 \% \mathrm{Cl}$ & 0.8423 & 0.8529 & 0.8421 \\
\hline$+95 \% \mathrm{Cl}$ & 0.9458 & 0.9423 & 0.9345 \\
\hline
\end{tabular}

and after extraction using DIAGNOdent, visual clinical examination, and bitewing radiographs. It turned out that visual evaluation as well as radiographs demonstrated higher specificity than laser fluorescence [12]. In the present research, the results obtained from the DIAGNOdent pen were closest to the visual and tactile assessment in case of ICDAS-II code 0 , indicating healthy enamel. However, in case of code 1 , most results were lower in the range of 0 - 13 . In case of more extended decay, DIAGNOdent pen codes 2 and 3 were the least convergent with the visual and tactile assessment, while the prevalent part of the results was higher than that reflected in the visual and tactile examination. In a study 


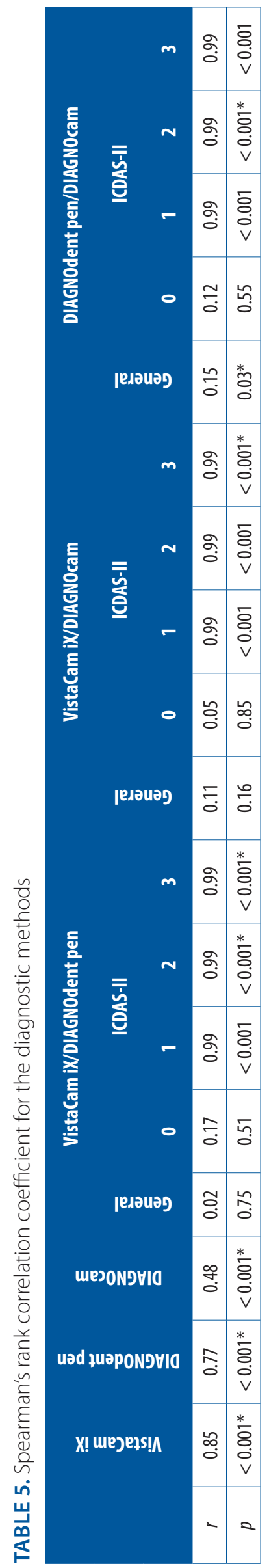

by Sheehy et al., DIAGNOdent repeatedly showed higher results in case of white decay spots. On the basis of results obtained from the assessment of occlusal surfaces decay, the authors emphasized that DIAGNOdent should be applied in combination with the visual examination, as it was not possible to distinguish between decalcification and caries with using one technique only [13]. In the present research, in the assessment of all material of the study, a full range of possible DIAGNOdent pen values was acquired from 0 to 99 . Lussi et al. [10] also obtained large divergences of their results. Various authors stated that the best compatibility between the DIAGNOdent examination and the clinical one was obtained in case of dentin caries $[14,15]$. In the present study, enamel decay was diagnosed; therefore, it was not possible to compare it with diagnostics of dentine decay. Sensitivity and specificity in this evaluation of the applied devices were related to the visual and tactile tooth assessment, which was adopted as a standard for the diagnostic evaluation test based on ICDAS-II. Marczuk-Kolada et al., in an ex vivo study concerning performance of ICDAS-II and fluorescence methods for detection of occlusal caries, used cone beam computed tomography (CBCT) to verify the extent of carious lesions [7]. In other authors' research, in evaluating the effectiveness of different diagnostic methods, histological examination of the hard dental substance of removed teeth or opening dental fissures was used. In the present study, this would not be possible due to ethical reasons, as the study focused on early caries without indications for opening the cavity and mechanical preparation. Moreover, in vitro experiment was not taken into consideration. In our research, the DIAGNOdent demonstrated lower sensitivity (64\%) than in other studies, such as in Lussi et al. (92\%) [10], Abalos et al. (89\%) [17], or Iranzo-Cortes (85\%) [16], whereas the device specificity was higher $(90 \%)$ than in the above authors' research. However, this present study focused only on enamel caries, while the others also considered dentine caries.

\section{CARIES DIAGNOSTICS WITH THE USE OF VISTACAM IX CAMERA}

In the available literature, there are very few studies on the use of fluorescence cameras for the detection of caries on occlusal surfaces. Most of them are based on in vitro or ex vivo research $[7-9,18]$, and only few of them are in vivo studies [19]. Jabłoński-Momeni et al. evaluated the reproducibility and precision of this method in caries detection. The study was conducted by two people: an experienced dental practitioner and a student, who with VistaProof camera evaluated occlusal surfaces of 53 extracted teeth. The camera showed high reproducibility of the results, regardless of the researchers' experience, and high accuracy in the detection of different stages of caries on occlusal surfaces [9]. 
In a different in vitro study, Jabłoński-Momeni et al. assessed occlusal surfaces caries using 4 methods, such as clinical study (ICDAS-II), DIAGNOdent laser fluorescence, VistaCam iX fluorescence camera, and bitewing photos. The results of clinical study and fluorescence camera were most similar to the gold standard, i.e. true stage of the caries. On the other hand, radiographs showed the least diagnostic value in the detection of occlusal surfaces' caries [18]. In yet another study, Jabłoński-Momeni compared the use of VistaProof and VistaCam iX cameras in the diagnostics of caries on occlusal surfaces. The study was conducted in vitro by two researchers: an experienced dental practitioner and a graduating dental student. Both the fluorescence cameras showed high reproducibility of the results and high accuracy of the detection of caries in different stages. Both the doctor and the student had no difficulties with software handling. The accuracy of both devices was comparable, with a slight prevalence of VistaProof [8]. In the present research, the results obtained with the VistaCam iX were more similar to the visual and tactile examination in case of ICDAS-II 1 and 3 codes, whereas in case of ICDAS-II code 0 , only some results were higher in relation to the visual and tactile examination, and were within $>0.9-1.5$ range signifying early caries. In case of ICDAS-II code 2, the VistaCam iX results were more compatible with the visual and tactile assessment, while the majority of results were higher than those obtained from the visual and tactile examination. The most sensitive method was the diagnostics with the VistaCam iX, with sensitivity amounting to $97 \%$. Also, the specificity of this method was very high, showing $90 \%$. In other studies, such as Rodrigues or Achilleos, the QLF method's sensitivity was also high, but the specificity was lower $[20,21]$.

\section{CARIES DIAGNOSTICS WITH THE USE OF DIGITAL TRANS-ILLUMINATION DIFOTI}

The digital transillumination DIFOTI was the least examined method of caries diagnostics, and a big obstacle in comparing its accuracy was the lack of recommended numeric scale, which would objectivize to a certain extent our study results. Since the results could not be quantitatively classified, the doctors had to conduct their own analysis, relying on their experience and considering the risk of their own errors. Moreover, it was not possible to evaluate the depth of the carious lesions [22]. In this research, an individual scale was created according to the grade of the tooth's obscuration, which helped to classify the obtained results. So far, very few experiments were conducted with the use of digital transillumination method, mainly in vitro $[23,24]$ and several in vivo $[25,26]$. The authors state, that DIFOTI method is efficient, especially in the detection of caries on proximal surfaces. The DIFOTI technique is often compared to radiological method, and may become its future alternative. With the use of DIFOTI method, early caries stages can be detected, which is not possible with the use of radiographs, where the lesion becomes visible only when extended to one third of the internal dentin part. However, the higher sensitivity of DIFOTI technique may sometimes lead to false diagnosis and restorative treatment recommendation, because white carious spots may be incorrectly interpreted as obscuration in the photograph from the camera, when related to healthy enamel. For this reason, transillumination method should be used to complement clinical examination [27]. In this research, the results obtained with the DIAGNOcam camera were mainly similar to the visual and tactile evaluation, when compared to those obtained with the VistaCam iX and DIAGNOdent. The results were mostly consistent as related to ICDAS-II codes 0 and 3, and less in case of ICDAS-II codes1 and 2, marking the existence of a carious spot. In an area of premolar teeth, where only proximal surfaces were examined with the device, the result was $3.92 \%$, and in case of molar permanent and deciduous teeth, where mainly occlusal surfaces were examined, the results were lower and accounted to $2.54 \%$ for permanent and $2.59 \%$ for deciduous molars. Söchtig et al. conducted a research with 130 patients, aged more than 12 , with a full set of permanent teeth. This research included visual and tactile evaluation as well as DIAGNOcam examination, and in justified cases, bitewing radiographs were taken. 85 patients were diagnosed with 127 lesions on proximal surfaces. It was stated that with the trans-illumination method, it is possible to detect caries on both proximal and occlusal surfaces. According to the authors, this method is a future alternative to bitewing radiographs; however, further study in this field is required [25]. Ástvaldsdóttir et al. compared diagnostic accuracy (effectiveness) of DIFOTI method in relation to conventional and digital radiography in detecting caries on proximal surfaces. The results showed that under in vitro conditions, the diagnostic accuracy of DIFOTI was better than that of radiological methods, in case of lesions on the occlusal surfaces affecting the enamel. In case of more extended caries affecting the dentin, the efficacy of all three methods were at the same level [23]. The DIFOTI method's sensitivity in this research amounted to $87 \%$ and specificity to $90 \%$. Vaarkamp et al. conducted a research on the diagnostic efficacy of bitewing radiographs and transillumination method in the detection of caries on proximal surfaces. They demonstrated that both the methods showed similar specificity (0.99-1.00); however, the sensitivity of DIFOTI method was significantly lower $(0.50-0.70)$ when compared to X-ray photos (0.71-1.00) [28]. Maia et al. evaluated the efficacy of trans-illumination and radiographs in diagnostics of early enamel caries on occlusal surfaces. The sensitivity of trans-illumination method reached 0.88 and the specificity amounted to 0.72 . As for the bitewing photos the sensitivity ranged from 0.35 to 0.53 , and the specificity from 0.50 to 0.72 . Enamel caries was better detectable using trans-illumination method rather than 
with radiograms [29]. Nowadays, there are a lot of devices available for the detection of dental caries. However, one must remember that they all have their advantages and disadvantages; therefore, they should be used in addition to general methods, which for many years have served as a basis for clinical detection of caries. However, if additional devices are selected, it is advisable to decide on two complementary approaches, e.g., DIFOTI with QLF [30]. A huge advantage is the possibility to monitor carious lesions, especially using the DIAGNOdent pen and VistaCam iX camera, because of numerical scales created for them. Previous reports concerning the application of new diagnostic devices, due to diverging opinions of the authors, require further research. According to the authors of this study, the use of the VistaCam iX and the DIAGNOcam cameras provided more reliable results than the DIAGNOdent pen, which showed large divergences and over-estimation of the results, particularly in case of proximal surfaces. As far as the suitability of the devices in dental practice is concerned, the DIAGNOcam technique is considered to be the most useful and may constitute a non-invasive alternative to the X-rays. Additionally, it facilitates the diagnostics of proximal surfaces, especially hard to reach in the visual and tactile examination. However, during an examination, the VistaCam iX method can be considered as more user-friendly and more pleasant for the patient, but it does not detect decay on the proximal surfaces. It is advisable to select each of the devices individually for the patient, depending on diagnostic needs. The study was conducted with the prior approval from the Bioethics Committee of Medical University of Silesia, December $16^{\text {th }}, 2014$, resolution No.: KNW/0022/KB1/99/I/14. The study was funded under contract number of KNW-2-047/D/5/N.

\section{CONCLUSIONS}

The basic method in caries diagnostics should be the clinical visual and tactile examination, which should be verified by applying an additional tool, such as the VistaCam iX and DIAGNOcam cameras or DIAGNOdent pen, since none of the devices, when used individually, can ensure full effectiveness in early caries detection. All of the evaluated devices are characterized by high specificity for caries detection; however, both the cameras show a higher level of sensitivity for the detection of carious lesions comparing to the DIAGNOdent pen.

\section{CONFLICT OF INTEREST}

The authors declare no potential conflicts of interest with respect to the research, authorship, and/or publication of this article.

\section{References}

1. Tranaeus S, Shi HQ, Angmar-Mansson B. Caries risk assessment: methods available to clinicians for caries detection. Community Dent Oral Epidemiol 2005; 33: 265-273.

2. Braga MM, Mendes FM, Ekstrand K. Detection activity assessment and diagnosis of dental caries lesions. Dent Clin N Am 2010; 54: 479-493.

3. Ismail AI, Sohn W, Tellez M, et al. The International Caries Detection and Assessment System (ICDAS): an integrated system for measuring dental caries. Community Dent Oral Epidemiol 2007; 35: 170-178.

4. Wenzel A. Bitewing and digital bitewing radiography for detection of caries lesions. J Dent Res 2004; 83: C72-C75.

5. Gomez J, Zakian C, Salsone S, et al. In vitro performance of different methods in detecting occlusal caries lesions. J Dent 2013; 41: 180-186

6. Luczaj-Cepowicz E, Marczuk-Kolada G, Obidzinska M, Sidun J. Diagnostic validity of the use of ICDAS II and DIAGNOdent pen verified by micro-computed tomography for the detection of occlusal caries lesions an in vitro evaluation. Lasers Med Sci 2019; 34: 1655-1663.

7. Marczuk-Kolada G, Luczaj-Cepowicz E, Obidzinska M, Rozycki J. Performance of ICDAS II and fluorescence methods on detection of occlusal caries - an ex vivo study. Photodiagnosis Photodyn Ther 2020; 29: 101609.

8. Jablonski-Momeni A, Liebegall F, Stoll R, Heinzel-Gutenbrunner M, Pieper K. Performance of a new fluorescence camera for detection of occlusal caries in vitro. Lasers Med Sci 2013; 28: 101-109.

9. Jablonski-Momeni A, Schipper HM, Rosen SM, et al. Performance of a fluorescence camera for detection of occlusal caries in vitro. Odontology 2011; 99: 55-61.

10. Lussi A, Megert B, Longbottom C, Reich E, Francescut P. Clinical performance of laser fluorescence device for detection of occlusal caries lessions. Eur J Oral Sci 2001; 109: 14-19.

11. Shi XQ, Welander U, Angmar-Mansson B. Occlusal caries detection with KaVo DIAGNOdent and radiography: an in vitro comparison. Caries Res 2000; 34: 151-158.

12. Angnes V, Angnes G, Batisttella M, Grande RHM, Loguercio AD, Reis A. Clinical effectiveness of laser fluorescence, visual inspection and radiography in the detection of occlusal caries. Caries Res 2005; 39: 490-495.

13. Sheehy EC, Brailsford SR, Kidd EA, Beighton D, Zoitopoulos L. Comparison between visual examination and a laser fluorescence system for in vivo diagnosis of occlusal caries. Caries Res 2001; 35: 421-426.

14. Wędrychowicz-Welman A, Prymas A, Kręgielczak A, NapionteKubanek H, Stopa BJ. Laser fluorescence technique for occlusal caries diagnosis. Polish J Environ Stud 2007; 16: 291-295.

15. Reis A, Mendes FM, Angnes V, Angnes G, Grande RH, Loguercio AD. Performance of methods of occlusal caries detection in permanent teeth under clinical and laboratory conditions. J Dent 2006; 34: 89-96.

16. Iranzo-Cortés JE, Terzic S, Montiel-Company JM, et al. Diagnostic validity of ICDAS and DIAGNOdent combined: an in vitro study in pre-cavitated lesions. Lasers Med Sci 2017; 32: 543-548.

17. Abalos C, Herrera M, Jiménez-Planas A, Llamas R. Performance of laser fluorescence for detection of occlusal dentinal caries lesions in permanent molars: an in vivo study with total validation of the sample. Caries Res 2009; 43: 137-141.

18. Jablonski-Momeni A, Stucke J, Steinberg T, Heinzel-Gutenbrunner. Use of ICDAS-II, fluorescence-based methods, and radiography in detection and treatment decision of occlusal caries lesions: an in vitro study. Int J Dent 2012; 2012: 371595.

19. Kockanat A, Unal M. In vivo and in vitro comparison of ICDAS II, DIAGNOdent pen, CarieScan PRO and SoproLife camera for occlusal caries detection in primary molar teeth. Eur J Paediatr Dent 2017; 18: 99-104. 
20. Rodrigues JA, Hug I, Diniz MB, Lussi A. Performance of fluorescence methods, radiographic examination and ICDAS II on occlusal surfaces in vitro. Caries Res 2008; 42: 297-304.

21. Achilleos EE, et al. Evaluation of a new fluorescence-based device in the detection of incipient occlusal caries lesions. Lasers Med Sci 2013; 28: 193-201.

22. Pretty IA, Maupome G. A closer look at diagnosis in clinical dental practice: part 5. Emerging technologies for caries detection and diagnosis. J Can Dent Assoc 2004; 70: 540, 540a-540i.

23. Ástvaldsdóttir Á, Åhlund K, Holbrook WP, De Verdier B, Tranæu S. Approximal caries detection by difoti: in vitro comparison of diagnostic accuracy/efficacy with film and digital radiography. Int J Dent 2012; 2012: 326401.

24. Young DA, Featherstone JD. Digital imaging fiber-optic transillumination, F-speed radiographic film and depth of approximal lesions. J Am Dent Assoc 2005; 136: 1682-1687.

25. Söchtig F, Hickel R, Kühnisch J. Caries detection and diagnostics with near-infrared light transillumination: clinical experiences. Quintessence Int 2014; 45: 531-538.

26. Bin-Shuwaish M, Yaman P, Dennison J, Neiva G. The correlation of DIFOTI to clinical and radiographic images in Class II carious lesions. Am Dent Assoc 2008; 139: 1374-1381.

27. Devlin H. Operative dentistry: a practical guide to recent innovations. Berlin Heidelberg: Springer; 2006.

28. Vaarkamp J, Bosch JJ, Verdonschot EH, Bronkhorst EM. The real performance of bitewing radiography and fiber-optic transillumination in approximal caries diagnosis. J Dent Res 2000; 79: 1757 1751.

29. Maia AMA, Karlsson L, Margulis W, Gomes A. Evaluation of two imaging techniques: near-infrared transillumination and dental radiographs for the detection of early approximal enamel caries. Dentomaxillofac Radiol 2011; 40: 429-433.

30. Gutierrez C. DIFOTI (Digital Fiberoptic Transillumination): Validität in Vitro. Dissertation, LMU München: Faculty of Medicine, 2009. 